\title{
The Radio Properties of BL Lac Objects
}

\author{
M. F. Aller, H. D. Aller, \& P. A. Hughes \\ University of Michigan, Ann Arbor, MI, U.S.A.
}

Abstract. The $\mathrm{cm}-\lambda$ properties of BL Lac objects are discussed.

Since 1979 we have systematically observed a flux-limited sample of BL Lac objects in order to quantify the cm-wavelength variability properties of this class of AGNs and to place constraints on the physical conditions in their parsec-scale flows by detailed modeling. The sample consists of 41 ob jects meeting the Hewitt \& Burbidge (1980) classification criteria with flux and declination limits $\mathrm{S}>0.4$ Jy at $8 \mathrm{GHz}$ and $-36^{\circ} \leq \delta \leq 81^{\circ}$ respectively.

We find that all program objects exhibited variations in total flux at our three observing frequencies, $14.5,8.0$, and $4.8 \mathrm{GHz}$; these flux changes exceed those for the QSOs in the Pearson-Readhead sample, a flux-limited group observed for a comparable time-period. The range and mean of a variability index which measures the peak-to-trough amplitude change (Aller, Aller, \& Hughes 1992 ) at $14.5 \mathrm{GHz}$ are: $0.20 \leq V \leq 0.91, V_{a v}=0.59$ for the BL Lacs and $0.20 \leq V \leq 0.72, V_{a v}=0.41$ for the 28 QSOs. These variations range from discrete events, with characteristic timescales as short as 0.26 years in the source frame for $0235+164$, to rises or decays lasting for many years on which some structure may be superimposed. VLBI studies show that BL Lacs may have many jet components, each lasting for about a decade (Gabuzda et al. 1994c), and thus the light curves can be complex. Almost all of the program objects also exhibited variations in polarized flux, and while in the case of QSOs we often find long-term preferred position angle orientations in our monitoring data, only 2 BL Lacs showed PA stability over the time-period of the study. Such temporal flux and polarization variations have been successfully modeled in a few cases by a shock-in-jet model (Hughes, Aller, \& Aller 1991); in others we can identify the signature of the passage of a shock, namely an increase in percentage polarization and a position angle swing. The best case remains BL Lac itself. Here individual outbursts match the development of new VLBI components (Denn \& Mutel, these Proceedings, p. 169). In a few additional cases VLBP maps have been available at crucial epochs (e.g., 0735+178, Gabuzda et al. 1994b) which support the interpretation that the changes result from the development of shocks at these times. In other cases we can identify a dominant transverse magnetic field from the PA data and a knowledge of the flow direction from the VLBI morphology which is consistent with the development of a transverse shock. As an example, in Figure 1 we show results for $1308+326$ which exhibited a preferred magnetic field orientation perpendicular to the flow direction in the mid-1980s. Unfortunately, no VLBP maps are available during this epoch of intense activity in both total and polarized flux when the source was partially opaque. Such behavior contrasts with that in the late 1980 s when the source was nearly transparent and the magnetic field was aligned along the flow direction. A plausible explanation for the frequent development of shocks in the parsec-scale flows of BL Lacs comes from the hydrodynamic simulations of e.g., Duncan \& Hughes (1994); in the slower flows, associated with BL Lacs 
from VLBI results, Kelvin-Helmhotz instabilities can occur readily leading to the development of internal shocks.

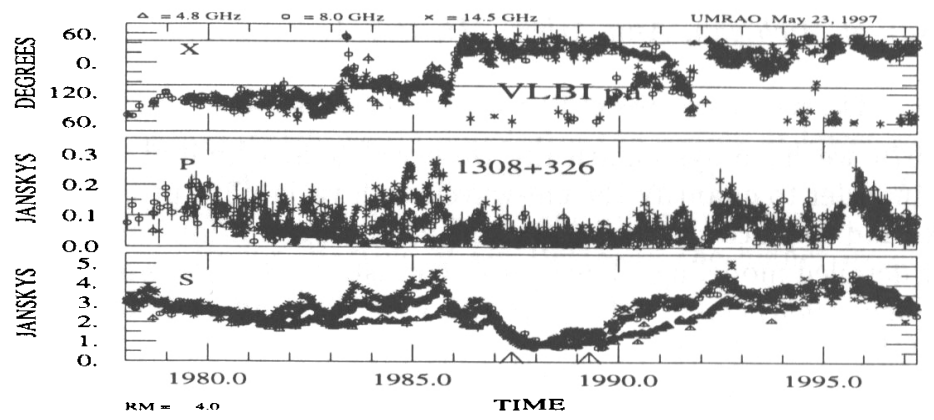

Figure 1. Daily averages of total flux density, polarized flux and PA for $1308+326$. Horizontal lines in the top panel mark the orientation of the VLBI structural axis (lower) and the direction orthogonal to it (upper). Arrows along the abscissa mark epochs of published VLBP maps (Gabuzda et al. 1994a).

OJ 287 is a very special class member. The optical variability suggests that the source is a binary black hole (Sillanpää et al. 1988) while the VLBI study of Cawthorne \& Wardle (1988) suggests that the viewing angle is small and near to the critical cone. Our data show nearly continuous activity with a particularly intense period in the mid to late $1980 \mathrm{~s}$. We have recently carried out a Morlet wavelet analysis of the flux and polarization which shows evidence for two periodicities: a stable modulation of 1.66 years associated with the underlying flow, and a superimposed signal of 1.12 years which we associate with the passage of a shock. The different periodicities of these two flow components may reflect different internal conditions in the flow domains, leading to different wave speeds, or different contractions of a single underlying periodicity, due to different Doppler factors in the two flow components.

Acknowledgments. We gratefully acknowledge support from NSF grant AST-9421979.

\section{References}

Aller, M. F., Aller, H. D., \& Hughes, P. A. 1992. ApJ, 399, 16-28.

Cawthorne, T. V., \& Wardle, J. F. C. 1988. ApJ, 332, 696-701.

Duncan, G. C., \& Hughes, P. A. 1994. ApJ, 436, L119-122.

Gabuzda, D. C., et al. 1994a. $A p J, 410,39-43$.

Gabuzda, D. C., et al. 1994b. ApJ, 435, 128-139.

Gabuzda, D. C., et al. 1994c. ApJ, 435, 140-161.

Hewitt, A., \& Burbidge, G. 1980. ApJS, 43, 57-158.

Hughes, P. A., Aller, H. D., \& Aller, M. F. 1991. ApJ, 374, 57-71.

Sillanpää, A., et al. 1988. ApJ, 325, 628-634. 